\title{
FACTORES LINGÜÍSTICOS Y SOCIALES EN EL PROCESO DE DEBILITAMIENTO DE /-N/ FINAL DE PALABRA Y EL USO DE FORMAS VULGARES EN LA CIUDAD DE MÁLAGA*
}

\author{
LINGUISTIC AND SOCIAL FACTORS OF THE /N/ SEGMENT IN FINAL POSITION'S \\ WEAKENING PROCESS AND THE USE OF VULGARISMS IN THE CITY OF MALAGA
}

\author{
Miriam Martín Morales \\ Universidad de Málaga \\ miriamartinmorales@gmail.com
}

Enviado: 29/08/2017

Aceptado: 08/05/2018

\begin{abstract}
Resumen
El objetivo de este trabajo es ofrecer los resultados de un estudio de carácter exploratorio realizados sobre materiales del corpus PRESEEA-Málaga. Por un lado, se analiza el proceso de debilitamiento de $/ \mathrm{n} /$ en posición final de palabra y, por otro, la distribución social de un conjunto de lexías, normativamente consideradas como vulgarismos generalizados, en función de su alternancia con las formas del español estándar (como para / pa o luego / aluego, entre otros). En cuanto a la realización de la nasal en posición final de palabra, se observa que, en Málaga, el proceso de debilitamiento se encuentra asentado en la velarización de este segmento, ya que esta realización es prácticamente categórica en todos los contextos. Por
\end{abstract}

\begin{abstract}
This research focuses on showing the results obtained in an exploratory analysis carried out using materials from PRESEEAMalaga corpus. The weakening process of the $/ \mathrm{n} /$ segment in final position is analysed, as well as the social distribution of a lexical group, usually considered as general vulgarisms, regarding its alternation with forms from standard Spanish (e.g.: para / pa or luego / aluego, among others). Considering the realization of the nasal in final position, it is observed that, in Malaga, the weakening process is stable at the velarisation point, since this realization is virtually categorical in all contexts. On the other side, the social distribution of lexical forms analysed leads
\end{abstract}

* Los datos y resultados en los que se basa este estudio proceden de los proyectos de investigación denominados "Patrones sociolingüísticos del español de Málaga" (PASOS) y "Estudio Complementario de los patrones Sociolingüísticos del Español de Málaga” (ECOPASOS), financiados por la DGICYT (FFI2011-29189-C05-01 y FFI2015-68171-C5-1-P) y fondos FEDER.

Para citar este artículo / To cite this article: Martín Morales, Miriam (2018). Factores lingüísticos y sociales en el proceso de debilitamiento de /-n/ final de palabra y el uso de formas vulgares en la ciudad de Málaga. ELUA, 32: 237-262. doi: 10.14198/ELUA2018.32.11

Enlace / Link: http://dx.doi.org/10.14198/ELUA2018.32.11 
otro lado, la distribución social de las variables léxicas analizadas lleva a determinar la existencia de dos patrones diferenciados en función de la edad de los hablantes que las favorecen.

PALABRAS CLAVE: /n/ final, vulgarismos, variedad de Málaga, PRESEEA. to the determination of the existence of two differentiated patterns according to the age of the speakers that promotes it.

KEYWORDS: /n/ segment in final position, vulgarism, Malaga variety, PRESEEA.

\section{INTRODUCCIÓN}

La ciudad de Málaga se encuentra en el área lingüística del español meridional que se caracteriza por la presencia de rasgos fonéticos innovadores. El español de Málaga presenta, por tanto, procesos como la pérdida de /-s/ en final de sílaba (Vida, 2004), el debilitamiento de /x/ intervocálica (Zmijanac, 2012) o la elisión de las consonantes /-d-/ o /-r-/ intervocálicas, que provoca la aparición de formas tradicionalmente consideradas vulgarismos, como pa < para o na < nada (Narbona, Cano y Morillo, 2003: 212-218).

En este trabajo se analiza la distribución lingüística y social de formas variables sometidas a procesos similares. Por un lado, la situación de una variable fonética: la realización de /-n/ implosiva en posición final de palabra y, por otro, la distribución social de determinadas formas, consideradas vulgares, que proceden de los procesos de debilitamiento que sufren las consonantes intervocálicas (ej.: $p a$ o $n a$ ). El estudio de estas últimas se complementa con el análisis de otras formas vulgares que derivan de otros procesos diferentes, como la monoptongación (pos < pues o mu< $<$ my) o del reforzamiento fonético (aluego < luego o asín $<$ así)

La descripción de la distribución lingüística y social de las variables estudiadas en este trabajo pretende ser una aportación más al conjunto de resultados procedentes del análisis de los materiales del corpus oral del español hablado en la ciudad de Málaga, parte del Proyecto para el estudio sociolingüístico del español de España y América.

\section{ESTADO DE LA CUESTIÓN}

\subsection{Segmento fonológico /-n/}

Uno de los principales objetivos de este trabajo es el estudio de la articulación de las variantes de $/ \mathrm{n} / \mathrm{en}$ final de palabra.

Alvar (1972: 120-124) indica que la velarización de /-n/ final es común en Las Palmas. La pérdida de la /-n/ final con nasalización de la vocal anterior aparece en los hablantes menos cultos. En otras islas del Archipiélago, la nasal final se velariza, aunque a veces se pierde, según señalan Alvar (1966: 507-548), Trujillo (1980: 103-107) y Lorenzo (1976: 70). En las zonas estudiadas, aparece la variante alveolar ante contexto prevocálico.

Los factores lingüísticos que condicionan la variabilidad de /-n/ implosiva son: la posición, el contexto y el estatus gramatical (Samper Padilla, 1990: 217-238).

En cuanto al contexto, López Morales (1983: 109) afirma que ante consonante prevalece la asimilación, mientras que ante vocal la nasal final se articula como alveolar y velar. Ante pausa, en San Juan, se prefiere la variante velar, seguida de la realización alveolar (Samper Padilla, 1990: 225). La pérdida de la nasal final está favorecida por el contexto prepausal, en contraste con el contacto con consonante. 
El estudio de Poplack (1979: 129-143) sobre el dialecto puertorriqueño de Filadelfia muestra que las variantes asimiladas presentan los porcentajes más altos ante consonante, pero en otros contextos predomina la velarización. La elisión se produce ante consonante más que ante vocal y ante pausa. Ma y Herasimchuk (1972: 349-464) estudian otro dialecto puertorriqueño, el de Jersey City, y observan que la velar es mayoritaria ante vocal. Sin embargo, ante consonante, predominan la velarización y la elisión.

Los datos de Terrell (1975: 257-271) en La Habana muestran que el proceso de elisión está avanzado, aunque la asimilación predomina ante consonante. Sin embargo, ante vocal y pausa, destaca la velar.

En el estudio de Hammond sobre el dialecto cubano de Miami (1979: 19-36) se comprueba que en el contexto preconsonántico y prepausal la articulación mayoritaria es la velar.

Los datos de Haché (1982: 145-154) sobre Santiago de los Caballeros señalan que en el contexto preconsonántico se prefiere la velar, seguida de la elisión. Ante vocal, aunque aumentan las alveolares, quedan lejos de las velares y de las elisiones. Ante pausa, predomina la velarización.

Según Cedergren (1973), la elisión de /-n/ en Panamá destaca ante consonante. Las articulaciones asimiladas son escasas en el contexto preconsonántico y no hay realizaciones alveolares ante vocal ni ante pausa. La velarización es más frecuente en el contexto prevocálico. Las cifras de elisiones que presenta este estudio son las más altas del Caribe. Esta afirmación aparece recogida por López Morales (1983: 109-111) al realizar una comparativa de los resultados obtenidos en diferentes trabajos.

En la tabla 1 se observa un resumen de la distribución de las realizaciones de /-n/ según el contexto lingüístico posterior. Los huecos en blanco se deben a la ausencia de datos sobre esos contextos.

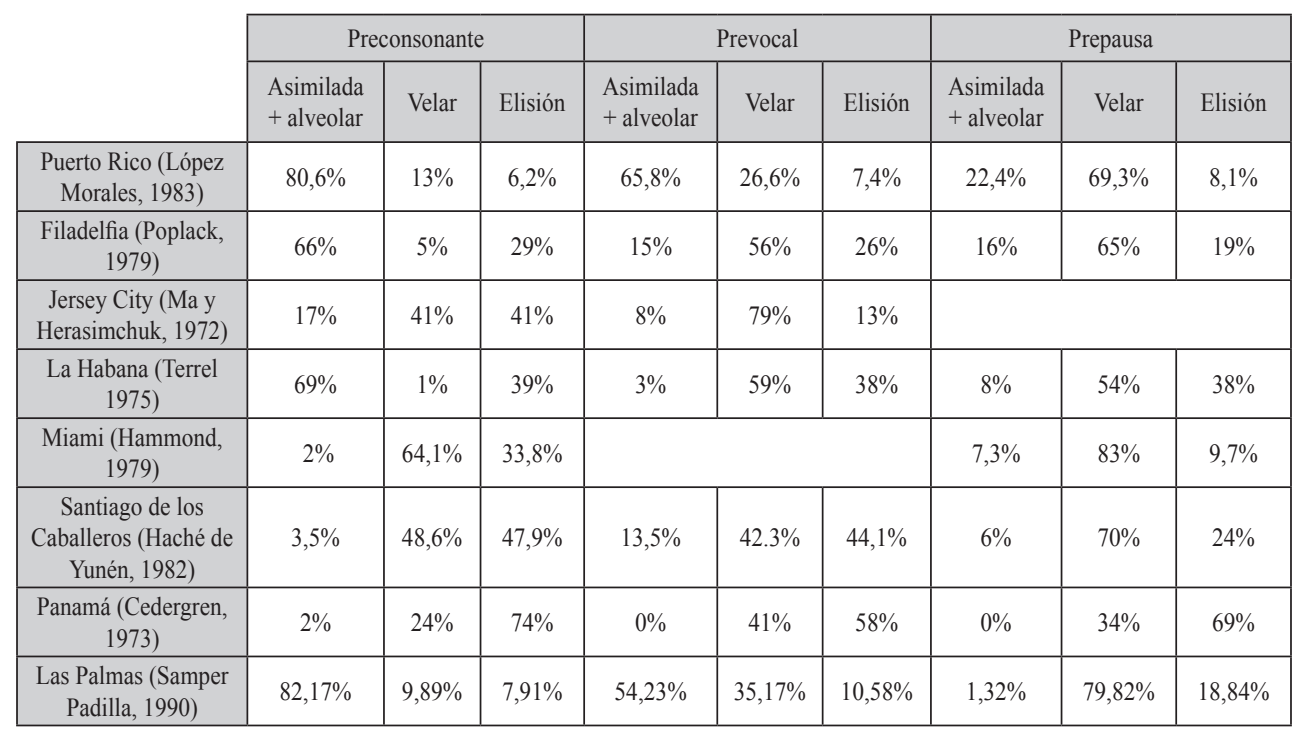

Tabla 1. Distribución de las realizaciones de /-n/ en posición final según el entorno posterior. 
La investigación de Poplack (1979: 129-143) y el estudio de Samper Padilla (1990: 229238) coinciden en que la pérdida de la nasalidad vocálica está relacionada con el carácter gramatical de /-n/. Este último afirmó que la elisión de /-n/ se produce siempre que esta repite una información aportada por otros elementos.

Terrell (1975: 257-271) y López Morales (1983: 109) propusieron diferentes reglas para el proceso de debilitamiento de la nasal. En primer lugar, afirmaron que la nasal se asimila a la consonante inicial de la palabra siguiente y que se velariza ante vocal y pausa. En cuanto a la velarización, López Morales (1983: 116) y Samper Padilla (1990 : 243) consideran que los contextos prepausal y prevocálico favorecen esta articulación.

De este modo, López Morales estableció los pasos del proceso de debilitamiento de la nasal (1983: 112-113):
I. asimilación consonántica;
II. debilitamiento ante vocal y pausa;
III. velarización incipiente que llega a convertirse en regla categórica;
IV. elisión de la nasal.

En cuanto a la regla de elisión, Cedergen (1973) demostró que en Panamá el contexto preconsonántico es el que más propicia la elisión en posición final. Sin embargo, Poplack (1979: 129-143) encontró en Filadelfia que la elisión predomina en contexto prepausal.

Más recientemente, Hauser (2012), analizó en profundidad el debilitamiento y la pérdida de la nasal en posición implosiva en las ciudades de Granada y Sevilla, distinguiendo entre sílaba final tónica y átona, partiendo de la hipótesis de que la zona oriental predominaba la velar en ambos contextos.

Con respecto a los factores sociales que favorece cada variable, se observa que, en Puerto Rico (López Morales, 1983: 117-120), la elisión aumenta entre los hablantes de niveles socioculturales bajos, mientras que la velarización es propia de los hablantes más jóvenes con niveles educaciones altos; en Las Palmas (Samper Padilla, 1990: 251-255), la elisión es propia de mujeres mayores con bajo nivel cultural, mientras que la velarización es un fenómeno propio de los hablantes más jóvenes.

\subsection{Vulgarismos}

Junto al proceso de velarización y elisión de /-n/ final, en este trabajo se estudia la realización variable de una serie de lexías que presentan realizaciones alternativas, fruto de determinados procesos fonéticos de reducción, monoptongación o refuerzo y que suelen ser considerados como vulgarismos.

I. La variable nada (adverbio o pronombre indefinido), que puede presentarse como $n a$.

II. El debilitamiento y pérdida de /-r-/ en la preposición para, que da lugar a pa.

III. Los diptongos /we/ y /ui/ en la conjunción pues y en el adverbio muy. En el caso de /we/ se contrae y presenta las formas pue, pos y po. En el caso de /ui/, el adverbio presenta la apócope de /-y/, articulándose como $m u$.

Asimismo, se analizan otras dos variables: luego y así, que pueden realizarse como aluego y asín. En el caso del adverbio luego se observa una prótesis al añadirse la vocal /a/ 
al principio de palabra. En así, se produce una paragoge, ya que la consonante /-n/ se añade al final de palabra. La variable así aparece recogida en el Atlas lingüistico y etnográfico de Castilla-La Mancha (AleCMan) (García y Moreno, 2003). En él, se observa una clara predominancia de la variante estándar así.

Las síncopas de nada en na y de para en pa se pueden relacionar con los procesos de debilitamiento y pérdida de /-d-/ y /-r-/ en posición intervocálica en español, estudiados ambos por Blanco (2004: 122-141) en Alcalá de Henares y López Morales (1983: 123-143) en Puerto Rico y el primero por Villena y Moya (2016: 281-321) en las ciudades de Granada y Málaga y Samper Padilla en Las Palmas (1990: 257-282).

En Andalucía, según Salvador (1964: 183-188), la pérdida de /-d-/ ya era común durante el S. XVIII, de ahí que esta región sea la que muestre el proceso en un estado más avanzado e incluso pueda considerarse un rasgo dialectal. Es más, Moya et alii (2012: 95-148) señalan que desde principios del siglo XV se ha podido observar un proceso de elisión de /-d-/ procedente de la /-t-/ latina, especialmente en desinencias verbales. Estos autores afirman que la ciudad de Málaga tiende hacia un patrón de estratificación en U. Asimismo, Villena y Moya (2016: 281321) estudiaron en profundidad la elisión de la dental en las ciudades de Málaga y Granada. En esta última, Moya et alii (2012: 95-148) mencionan que, la variable nada, junto con todo, representan más del $80 \%$ de casos de elisión. Aunque estos autores no recogen diferencias significativas en cuanto al género, la elisión suele aparecer en jóvenes de nivel educacional bajo.

En cuanto al debilitamiento de /-r-/ en la preposición para, en el estudio de Blanco (2004: 138-141) se observa que la conservación de la vibrante es la realización más común. La elisión absoluta ha sido relegada a los contextos informales y está favorecida por el nivel más bajo de instrucción. Esta autora también destacó la existencia de un patrón regular, puesto que a mayor nivel de estudios corresponde una mayor probabilidad de mantenimiento pleno de la /-r-/.

Las reducciones de los diptongos en las formas pues y muy también fueron analizadas por Blanco (2004: 107-110, 115-118) en Alcalá de Henares. Aunque sus resultados revelan que en esta ciudad predominan las realizaciones normativas, el uso de pos es más frecuente entre las generaciones más jóvenes independientemente de la edad o el grado de instrucción, $m u$, sin embargo, es una variante propia de hombres con bajo nivel de instrucción, pero también jóvenes.

\section{PREGUNTAS DE INVESTIGACIÓN}

Como ya se ha indicado en el epígrafe introductorio, el objetivo de este trabajo es, por un lado, describir cuál es la situación del fonema /-n/ en posición final de palabra con relación al proceso de velarización y elisión descrito en otros ámbitos del mundo hispánico y, por otra, analizar la distribución social de determinadas formas vulgares. En el primero de los casos, se pretende averiguar si, en Málaga, el proceso de elisión goza de alguna vitalidad y se puede dibujar algún tipo de estratificación o si, por el contrario, los hablantes de esta variedad optan por alguna de las realizaciones de manera categórica. En cuanto al uso de los vulgarismos, la intención es conocer si todas las formas analizadas siguen el mismo patrón y son favorecidas por los mismos grupos de hablantes (principalmente personas mayores con bajos niveles de instrucción) o si, en cambio, existen diferencias entre las distintas lexías y algunas de ellas se difunden, favorecidas por el hecho de proceder de procesos fonéticos de debilitamiento, entre las generaciones más jóvenes. 


\section{METODOLOGÍA}

\subsection{El proyecto PRESEEA y el corpus de Málaga}

Para comprobar la hipótesis planteada en el epígrafe 2, se han utilizados los materiales del Corpus Sociolingüístico de la ciudad de Málaga, incluido en el proyecto PRESEEA (Proyecto para el Estudio Sociolingüístico del Español de España y de América). Los fundamentos teóricos y metodológicos que sustentan el proyecto fueron expuestos por Francisco Moreno Fernández (1996), y están disponibles en la página web del proyecto PRESEEA.

Se trata de una colección de entrevistas semidirigidas realizadas a una muestra representativa de hablantes de la ciudad de Málaga (72 en total).

\subsection{Universo de la muestra}

La ciudad de Málaga se encuentra en la costa al sur de la Península Ibérica. Según el Padrón Municipal de Habitantes, en el momento de la recogida del corpus, la ciudad contaba con una población de 560.755 habitantes, de los cuales un 51.64\% son mujeres y un $48.35 \%$ son hombres.

\begin{tabular}{|c|c|c|c|c|c|c|c|c|c|c|}
\hline & \multicolumn{2}{|c|}{$\begin{array}{c}\text { Menores de } 20 \\
\text { años }\end{array}$} & \multicolumn{2}{|c|}{$\begin{array}{c}\text { Entre } 20 \text { y } 34 \\
\text { años } \\
\end{array}$} & \multicolumn{2}{|c|}{$\begin{array}{c}\text { Entre } 35 \text { y } 54 \\
\text { años }\end{array}$} & \multicolumn{2}{|c|}{$\begin{array}{l}\text { De } 55 \text { años en } \\
\text { adelante }\end{array}$} & \multicolumn{2}{|c|}{ Total } \\
\hline & $\mathbf{N}$ & $\%$ & $\mathbf{N}$ & $\%$ & $\mathbf{N}$ & $\%$ & $\mathbf{N}$ & $\%$ & $\mathbf{N}$ & $\%$ \\
\hline Hombres & 62367 & 11,2 & 72753 & 12,97 & 77211 & 13,76 & 58823 & 10,48 & 271154 & 48,35 \\
\hline Mujeres & 59292 & 10,57 & 69612 & 12,41 & 82348 & 14,68 & 78349 & 13,97 & 289601 & 51,64 \\
\hline Total & 121659 & 21,69 & 142365 & 25,38 & 159559 & 28,45 & 137172 & 24,46 & 560755 & 100 \\
\hline
\end{tabular}

Tabla 2. Población de la ciudad de Málaga en 2001.

\begin{tabular}{|c|c|c|c|c|c|c|c|c|c|c|c|}
\hline \multirow{2}{*}{ Sexo } & \multirow{2}{*}{ Edad } & \multicolumn{2}{|c|}{ Sin estudios } & \multicolumn{2}{|c|}{ Nivel elemental } & \multicolumn{2}{|c|}{ Nivel medio } & \multicolumn{2}{|c|}{ Nivel superior } & \multicolumn{2}{|c|}{ Total } \\
\hline & & $\mathbf{N}$ & $\%$ & $\mathbf{N}$ & $\%$ & $\mathbf{N}$ & $\%$ & $\mathbf{N}$ & $\%$ & $\mathbf{N}$ & $\%$ \\
\hline \multirow{4}{*}{ Hombres } & 20-34 & 1742 & 0,39 & 8098 & 1,84 & 45882 & 10,47 & 12628 & 2,88 & 68350 & 15,60 \\
\hline & $35-54$ & 5196 & 1,18 & 13461 & 3,07 & 37400 & 8,63 & 12793 & 2,92 & 68850 & 15,72 \\
\hline & $55-$ & 15868 & 3,62 & 13216 & 3,01 & 17068 & 3,89 & 5421 & 1,23 & 51573 & 11,77 \\
\hline & total & 22806 & 5,20 & 34775 & 7,94 & 100350 & 22,91 & 30842 & 7,04 & 188773 & 43,10 \\
\hline \multirow{4}{*}{ Mujeres } & 20-34 & 1483 & 0,33 & 6133 & 1,40 & 42484 & 9,70 & 17269 & 3,94 & 67369 & 15,38 \\
\hline & $35-54$ & 7736 & 1,76 & 15560 & 3,55 & 39562 & 9,03 & 12669 & 2,89 & 75527 & 17,24 \\
\hline & 55- & 66747 & 15,2 & 19288 & 4,40 & 17056 & 3,89 & 3203 & 0,73 & 106294 & 24,27 \\
\hline & total & 75966 & 17,34 & 40981 & 9,35 & 99102 & 22,62 & 3341 & 7,56 & 249190 & 56,89 \\
\hline \multicolumn{2}{|c|}{ Total } & 98772 & 22,55 & 75756 & 17,29 & 199452 & 45,54 & 63983 & 14,60 & 437963 & 100 \\
\hline
\end{tabular}

Tabla 3. Población de la ciudad de Málaga (2001) según el sexo y el nivel de estudios. 
Por otro lado, los datos extraídos del censo de población y viviendas de 2001 muestran que en Málaga predominaban las personas con estudios medios (45,54\%). Además, la ciudad contaba con un importante porcentaje de población carente de estudios $(22,55 \%)$ y con estudios elementales $(17,29 \%)$.

\subsection{Muestra de informantes}

Esta muestra se divide en tres grupos generacionales: informantes entre 20 y 34 años, sujetos entre 35 y 54 y la última generación formada por individuos de más de 55 años; los informantes también aparecen divididos según el sexo: hombres y mujeres, y en tres niveles de instrucción: primaria (0-10 años de escolarización), secundaria (unos 10-14 años de estudios) y superior (15 años de escolarización aproximadamente). El equipo PRESEEA-Málaga trabajó, además, con variables de post-estratificación que recogen información complementaria.

\begin{tabular}{|c|c|c|c|c|c|c|}
\hline & \multicolumn{2}{|c|}{$\begin{array}{c}\text { Generación 1 } \\
(20-34 \text { años) }\end{array}$} & \multicolumn{2}{c|}{$\begin{array}{c}\text { Generación 2 } \\
\text { (35-54 años) }\end{array}$} & \multicolumn{2}{c|}{$\begin{array}{c}\text { Generación 3 } \\
\text { (más de 55 años) }\end{array}$} \\
\cline { 2 - 7 } & Hombres & Mujeres & Hombres & Mujeres & Hombres & Mujeres \\
\hline $\begin{array}{c}\text { Nivel de } \\
\text { instrucción 1 }\end{array}$ & & & & & & \\
\hline $\begin{array}{c}\text { Nivel de } \\
\text { instrucción 2 }\end{array}$ & & & & & & \\
\hline $\begin{array}{c}\text { Nivel de } \\
\text { instrucción 3 }\end{array}$ & & & & & & \\
\hline
\end{tabular}

Tabla 4. Distribución de los informantes según el nivel de instrucción, la edad y el sexo

\subsection{Recogida y tratamiento de los datos}

La recogida de los materiales se realizó mediante la grabación de conversaciones semidirigidas. Las grabaciones fueron transliteradas mediante ortografía ordinaria y etiquetadas mediante las normas internacionales de marcación textual de la Text Encoding Initiative (TEI), siguiendo un sistema consensuado para todos los grupos PRESEEA (Vida, 2007: 37-66).

A partir de los materiales transliterados y etiquetados se procedió a la codificación de las variables. La discriminación de los diferentes alófonos se llevó a cabo de forma perceptiva por un mismo juez, aunque se llevó a cabo una prueba de control para comprobar que otros receptores codificaban los sonidos de la misma forma. El análisis estadístico se llevó a cabo con el programa SPSS (versión 21) para Mac.

En este estudio, las variables dependientes fueron la nasal implosiva (/-n/) en final de palabra y los procesos de debilitamiento y adición fonéticos en las variables luego, pues, para, así, muy y nada. En el caso de las variables léxicas se analizaron las grabaciones completas de los 72 informantes. Sin embargo, dado el número de ocurrencias de /-n/, se analizaron 6 minutos al azar. Las variantes analizadas de la nasal implosiva para este trabajo fueron las siguientes: 


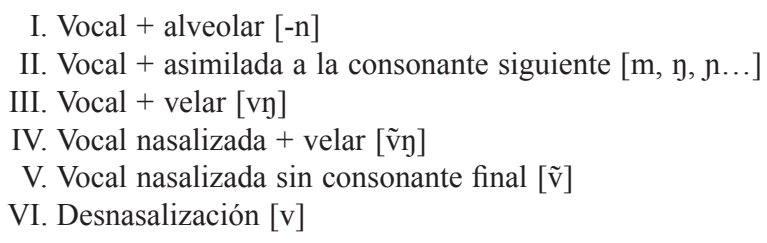

En cuanto a la variante luego, se analizaron las realizaciones luego y aluego. En pues se agruparon las realizaciones en pues y pue, por un lado, y pos pos y po, por otro. La variante para fue analizada junto con su variante $p a$. En el estudio de así se analizó su variante asín. Muy presentó la variante $m u$. Por último, en nada se observó la pérdida de la dental (na).

Para poder analizar los datos recopilados fue necesario aplicar la estadística inferencial. Con el fin de analizar la relación de las variables cualitativas se acudió a la prueba del chi-cuadrado $\left(\mathrm{X}^{2}\right)$. Según Fasold (1996: 155-175), esta prueba estadística determina la independencia de las variables. Para comprobar la significación de los resultados, se utilizó el umbral del 5\% de error establecido para los estudios sociales. Además, se obtuvieron también los valores de Phi y la V de Cramer, con el fin de observar la dependencia o independencia de las variables.

\section{RESULTADOS}

\subsection{Segmento fonológico /-n/}

El proceso de velarización y debilitamiento de la nasal final en Málaga se encuentra en una fase intermedia. Las variantes analizadas fueron recodificadas en:

I. Vocal + alveolar [-n] y vocal + asimilada a la consonante siguiente $[\mathrm{m}, \mathrm{y}, \mathrm{n} \ldots$.$] que, a partir$ de aquí, representaremos con la etiqueta alveolar o asimilada.

II. Vocal + velar [vy] y vocal nasalizada + velar [ṽ̃], que pasará a velarización.

III. Vocal nasalizada sin consonante final [̃̃] y desnasalización [v], de ahora en adelante elisión.

\begin{tabular}{|c|c|c|}
\cline { 2 - 3 } \multicolumn{1}{c|}{} & Frecuencia & Porcentaje \\
\hline Alveolar o asimilada & 42 & $1,1 \%$ \\
\hline Velar & 3672 & $95,5 \%$ \\
\hline Elisión & 132 & $3,4 \%$ \\
\hline Total & 3846 & $100 \%$ \\
\hline
\end{tabular}

Tabla 5. Frecuencias absolutas y relativas de las realizaciones de /-n/ tras la recodificación. 
Las realizaciones alveolares y asimiladas son las menos frecuentes $(1,1 \%)$, seguidas por pérdida del segmento nasal $(3,4 \%)$. La articulación velar es, por lo tanto, la más frecuente, con un $95,5 \%$.

A continuación, se analizó la influencia del entorno posterior en la variación del segmento /-n/ en final de palabra.

\begin{tabular}{|c|c|c|c|c|}
\cline { 2 - 5 } \multicolumn{1}{c|}{} & Vocal & Consonante & Pausa & Total \\
\hline \multirow{3}{*}{ Alveolar o asimilada } & 2 & 35 & 5 & 42 \\
\cline { 2 - 5 } & $0,2 \%$ & $1,5 \%$ & $1,1 \%$ & $1,1 \%$ \\
\hline \multirow{2}{*}{ Velar } & 1065 & 2210 & 397 & 3672 \\
\cline { 2 - 5 } & $96,8 \%$ & $95,9 \%$ & $90,0 \%$ & $95,5 \%$ \\
\hline \multirow{2}{*}{ Elisión } & 33 & 60 & 39 & 132 \\
\cline { 2 - 5 } & $3,0 \%$ & $2,6 \%$ & $8,8 \%$ & $3,4 \%$ \\
\hline \multirow{2}{*}{ Total } & 1100 & 2305 & $100 \%$ & $100 \%$ \\
\cline { 2 - 5 } & $100 \%$ & $100 \%$ & 1846 \\
\hline \multirow{5}{*}{ Coeficiente Chi-cuadrado de Pearson: $56,652(4)$. Sig.: $>, 001$} \\
V de Cramer: 0,086. Sig.: $>, 001$ \\
Phi: 0,121. Sig.: $>, 001$ \\
\hline
\end{tabular}

Tabla 6. Realización de /-n/ según el entorno posterior.

Frecuencias absolutas, relativas y significación estadística

Tal y como se comprueba en la tabla 6, el entorno prevocálico favorece la realización velar $(96,8 \%)$, aunque la diferencia es mínima con respecto al preconsonántico y al prepausal $(95,9 \%$ y $90 \%$, respectivamente). Por otro lado, el entorno prepausal favorece la elisión (8,8\%). Las diferencias entre los grupos se muestran estadísticamente significativas.

La tabla 6 también muestra que los resultados obtenidos en el test de $\mathrm{X}^{2}$ tienen una significación de $>, 001$, lo que indica que son muy significativos. Por otro lado, los resultados de la prueba de Phi y V de Cramer muestran que la relación es significativa $(>, 001)$, indicando que la probabilidad de error es mínima.

En la tabla 7 se presentan los resultados obtenidos tras analizar el efecto de la funcionalidad en la pérdida de /-n/ final. Para ello se han distinguido, por un lado, los casos en que la /-n/ es un morfema verbal de $3^{\text {a }}$ persona del plural (cantaN). En estos casos, esta forma se podría confundir con otras formas verbales (canta). Por otro lado, se distinguieron los casos en los que la /-n/ final es lexemática ( $p a N$, cancióN).

Como se puede observar, la elisión es más frecuente en aquellos casos en los que el segmento /-n/ tiene estatus gramatical (5,4\%). Este resultado está en línea con los de otras comunidades de habla, como Las Palmas (Samper Padilla, 1990: 229-238) o Filadelfia (Poplack, 1979: 129-143). Asimismo, se podría establecer una relación con los resultados encontrados en estudios para el proceso de elisión de /-s/ final (Vida, 2004: 132-139; López Morales, 1983: 47-48), favorecido también por el estatus gramatical de este segmento. Se apoya así, por tanto, la hipótesis contrafuncional (Samper Padilla, 1990: 229; Vida, 2004: 153). 


\begin{tabular}{|c|c|c|c|}
\hline & Morfemática & No morfemática & Total \\
\hline \multirow{2}{*}{ Alveolar o asimilada } & 13 & 29 & 42 \\
\hline & $1,3 \%$ & $1,0 \%$ & $1,1 \%$ \\
\hline \multirow{2}{*}{ Velar } & 923 & 2749 & 3672 \\
\hline & $93,3 \%$ & $96,2 \%$ & $95,5 \%$ \\
\hline \multirow{2}{*}{ Elisión } & 53 & 79 & 132 \\
\hline & $5,4 \%$ & $2,8 \%$ & $3,4 \%$ \\
\hline \multirow{2}{*}{ Total } & 989 & 2857 & 3846 \\
\hline & $100 \%$ & $100 \%$ & $100 \%$ \\
\hline \multicolumn{4}{|c|}{$\begin{array}{c}\text { Coeficiente Chi-cuadrado de Pearson: 15,649 (2). Sig.: >,001 } \\
\text { V de Cramer: 0,064. Sig.: >,001 } \\
\text { Phi: 0,064. Sig.: }>, 001\end{array}$} \\
\hline
\end{tabular}

Tabla 7. Realizaciones de /-n/ según la funcionalidad. Frecuencias absolutas, relativas y significación estadística

Con respecto a los factores extralingüísticos, se puede observar (tabla 8) que el cero fonético es más frecuente entre el grupo generacional de mayor edad (4,8\%).

\begin{tabular}{|c|c|c|c|c|}
\cline { 2 - 5 } \multicolumn{1}{c|}{} & $20-34$ años & $35-54$ años & +55 años & Total \\
\hline \multirow{2}{*}{ Alveolar o asimilada } & 11 & 11 & 20 & 42 \\
\cline { 2 - 5 } & $0,9 \%$ & $1,0 \%$ & $1,3 \%$ & $1,1 \%$ \\
\hline \multirow{2}{*}{ Velar } & 1148 & 1115 & 1409 & 3672 \\
\cline { 2 - 5 } & $96,6 \%$ & $96,5 \%$ & $93,9 \%$ & $95,5 \%$ \\
\hline \multirow{2}{*}{ Elisión } & 30 & 30 & 72 & 132 \\
\cline { 2 - 5 } & $2,5 \%$ & $2,6 \%$ & $4,8 \%$ & $3,4 \%$ \\
\hline \multirow{2}{*}{ Total } & 1189 & 1156 & 1501 & 3846 \\
\cline { 2 - 5 } & $100 \%$ & $100 \%$ & $100 \%$ & $100 \%$ \\
\hline \multirow{2}{*}{ Coeficiente Chi-cuadrado de Pearson: $15,338(4)$. Sig.:, 004} \\
V de Cramer: 0,045. Sig.: ,004 \\
\multicolumn{4}{c}{ Phi: 0,063. Sig.: ,004 } \\
\hline
\end{tabular}

Tabla 8. Realizaciones de /-n/ según la edad. Frecuencias absolutas, relativas y significación estadística

A continuación, se analizó la articulación de /-n/ según el nivel educativo de los informantes. Para esto, los informantes fueron divididos en: informantes sin estudios, informantes con estudios primarios, informantes con estudios secundarios e informantes con estudios superiores. A la luz de los resultados, se realizó una recodificación que pusiese de manifiesto la diferencia observada entre los diferentes niveles educativos (tabla 9). 


\begin{tabular}{|c|c|c|c|}
\hline & $\begin{array}{l}\text { Sin estudios } \\
\text { Estudios primarios } \\
\text { Secundaria }\end{array}$ & Estudios superiores & Total \\
\hline \multirow{2}{*}{ Alveolar o asimilada } & 39 & 3 & 42 \\
\hline & $1,7 \%$ & $0,2 \%$ & $1,1 \%$ \\
\hline \multirow{2}{*}{ Velar } & 2172 & 1500 & 3672 \\
\hline & $94,5 \%$ & $97,0 \%$ & $95,5 \%$ \\
\hline \multirow{2}{*}{ Elisión } & 88 & 44 & 132 \\
\hline & $3,8 \%$ & $2,8 \%$ & $3,4 \%$ \\
\hline \multirow{2}{*}{ Total } & 2299 & 1547 & 3846 \\
\hline & $100 \%$ & $100 \%$ & $100 \%$ \\
\hline \multicolumn{4}{|c|}{$\begin{array}{c}\text { Coeficiente Chi-cuadrado de Pearson: 22,321 (2). Sig.: >,001 } \\
\text { V de Cramer: 0,076. Sig.: >,001 } \\
\text { Phi: 0,076. Sig.: }>, 001\end{array}$} \\
\hline
\end{tabular}

Tabla 9. Realización de /-n/ según el nivel educativo. Frecuencias absolutas, relativas y significación estadística.

Los datos que aquí se presentan muestran que los informantes con un menor nivel de estudios son los que ofrecen un mayor número de ocurrencias de elisión (3,8 \%).

Con referencia al sexo, en la tabla 10 se presentan los datos obtenidos del análisis de la influencia del sexo en la realización de /-n/.

\begin{tabular}{|c|c|c|c|}
\hline & & & \\
\hline & Hombre & Mujer & Total \\
\hline \multirow{2}{*}{ Alveolar o asimilada } & 19 & 23 & 42 \\
\hline & $0,9 \%$ & $1,2 \%$ & $1,1 \%$ \\
\hline \multirow{2}{*}{ Velar } & 1899 & 1773 & 3672 \\
\hline & $94,9 \%$ & $96,1 \%$ & $95,5 \%$ \\
\hline \multirow{2}{*}{ Elisión } & 83 & 49 & 132 \\
\hline & $4,1 \%$ & $2,7 \%$ & $3,4 \%$ \\
\hline \multirow{2}{*}{ Total } & 2001 & 1845 & 3846 \\
\hline & $100 \%$ & $100 \%$ & $100 \%$ \\
\hline \multicolumn{4}{|c|}{$\begin{array}{c}\text { Coeficiente Chi-cuadrado de Pearson: 7,146 (2). Sig.: ,028 } \\
\text { V de Cramer: 0,043. Sig.: ,028 } \\
\text { Phi: 0,043. Sig.: ,028 }\end{array}$} \\
\hline
\end{tabular}

Tabla 10. Realización de /-n/ según el sexo. Frecuencias absolutas, relativas y significación estadística.

Se observa que los hombres presentan una ligera tendencia a la elisión de la consonante $(4,1 \%)$ frente a las mujeres $(2,7 \%)$. Lo contrario ocurre con la realización alveolar y asimilada, donde son las mujeres quienes tienden levemente a propiciarla con un $1,2 \%$ frente al $0,9 \%$ de los hombres. 
En resumen, se puede decir que, en la ciudad de Málaga, la articulación velar de la nasal implosiva en posición final de palabra es prácticamente categórica. La elisión del segmento nasal se ve favorecida por los hombres mayores, especialmente en posición prepausal y cuando es marca gramatical. Además, las relaciones entre las variables estudiadas y las diferentes realizaciones de la nasal parecer ser estadísticamente representativas.

\subsection{Vulgarismos como resultado de procesos de refuerzo fonético}

\subsubsection{Luego}

La realización de la variable luego alterna entre la forma estándar (luego) y la epentética aluego.

\begin{tabular}{|c|c|c|}
\cline { 2 - 3 } \multicolumn{1}{c|}{} & Frecuencia & Porcentaje \\
\hline Luego & 1064 & $97,9 \%$ \\
\hline Aluego & 23 & $2,1 \%$ \\
\hline Total & 1087 & $100 \%$ \\
\hline
\end{tabular}

Tabla 11. Frecuencias absolutas y relativas de las realizaciones de luego.

Como se puede observar en la tabla 11, la realización de la variable como luego es casi categórica con un 97,9\% de las ocurrencias, frente al 2,1\% de aluego.

\begin{tabular}{|c|c|c|c|}
\cline { 2 - 4 } \multicolumn{1}{c|}{} & Hombre & Mujer & Total \\
\hline \multirow{2}{*}{ Luego } & 382 & 682 & 1064 \\
\cline { 2 - 4 } & $95,0 \%$ & $99,6 \%$ & $97,9 \%$ \\
\hline \multirow{2}{*}{ Aluego } & 20 & 3 & 23 \\
\cline { 2 - 4 } & $5,0 \%$ & $0,4 \%$ & $2,1 \%$ \\
\hline \multirow{2}{*}{ Total } & 402 & 685 & 1087 \\
\cline { 2 - 4 } & $100 \%$ & $100 \%$ & $100 \%$ \\
\hline \multirow{2}{*}{ Coeficiente Chi-cuadrado de Pearson: $25,179(1)$. Sig.: $>, 001$} \\
V de Cramer: 0,152. Sig.: $>, 001$ \\
Phi: -0,152. Sig.: $>, 001$
\end{tabular}

Tabla 12. Realizaciones de luego según el sexo. Frecuencias absolutas, relativas y significación estadística.

Asimismo, se pensó que la edad podía influir a la hora de elegir entre luego y aluego.

Aunque la realización luego se mantiene predominante en todos los niveles, en la tabla 13 se muestra una leve tendencia a la realización de aluego a medida que aumenta la edad de los informantes $(2,8 \%)$. Se establece así una relación significativa entre la edad y las diferentes realizaciones. 


\begin{tabular}{|c|c|c|c|}
\cline { 2 - 4 } \multicolumn{1}{c|}{} & $20-34$ años & +35 años & Total \\
\hline \multirow{2}{*}{ Luego } & 331 & 733 & 1064 \\
\cline { 2 - 4 } & $99,4 \%$ & $97,2 \%$ & $97,9 \%$ \\
\hline \multirow{2}{*}{ Aluego } & 2 & 21 & 23 \\
\cline { 2 - 4 } & $0,6 \%$ & $2,8 \%$ & $2,1 \%$ \\
\hline \multirow{2}{*}{ Total } & 333 & 754 & 1087 \\
\cline { 2 - 4 } & $100 \%$ & $100 \%$ & $100 \%$ \\
\hline \multirow{2}{*}{ Coeficiente Chi-cuadrado de Pearson: $5,322(1)$. Sig.:, 021} \\
V de Cramer: 0,070. Sig.: , 021 \\
Phi: 0,070. Sig.: ,021
\end{tabular}

Tabla 13. Realizaciones de luego según la edad. Frecuencias absolutas, relativas y significación estadística.

\begin{tabular}{|c|c|c|c|}
\cline { 2 - 4 } \multicolumn{1}{c|}{} & $\begin{array}{c}\text { Sin estudios } \\
\text { Estudios primarios }\end{array}$ & $\begin{array}{c}\text { Secundaria } \\
\text { Estudios superiores }\end{array}$ & Total \\
\hline \multirow{2}{*}{ Luego } & 169 & 895 & 1064 \\
\cline { 2 - 4 } & $88,0 \%$ & $100,0 \%$ & $97,9 \%$ \\
\hline \multirow{2}{*}{ Aluego } & 23 & 0 & 23 \\
\cline { 2 - 4 } & $12,0 \%$ & $0,0 \%$ & $2,1 \%$ \\
\hline \multirow{2}{*}{ Total } & 192 & 895 & 1087 \\
\cline { 2 - 4 } & $100 \%$ & $100 \%$ & $100 \%$ \\
\hline \multirow{2}{*}{ Coeficiente Chi-cuadrado de Pearson: $109,531(1)$. Sig.: $>, 001$} \\
V de Cramer: 0,317. Sig.: $>, 001$ \\
Phi: -0,317. Sig.: >,001
\end{tabular}

Tabla 14. Realizaciones de luego según el nivel educativo.

Frecuencias absolutas, relativas y significación estadística.

En cuanto al nivel educativo, se observa (tabla 14) que la realización de aluego solo presenta ocurrencias en el caso de los informantes de niveles educativos bajos (12\%).

\subsubsection{Asi}

La forma así alterna con la variante asín. Las frecuencias aparecen en la tabla 15, donde se observa que la forma estándar es mayoritaria (91,2\%).

Cuando se analiza la influencia del sexo (tabla 16), se observa que la forma asín es favorecida por los hombres, que duplican el porcentaje que se puede observar en el caso de las mujeres. Como se puede observar, las diferencias son estadísticamente significativas. 


\begin{tabular}{|c|c|c|}
\cline { 2 - 3 } \multicolumn{1}{c|}{} & Frecuencia & Porcentaje \\
\hline Así & 930 & $91,2 \%$ \\
\hline Asín & 90 & $8,8 \%$ \\
\hline Total & 1020 & $100 \%$ \\
\hline
\end{tabular}

Tabla 15. Frecuencias absolutas y relativas de las realizaciones de así.

\begin{tabular}{|c|c|c|c|}
\cline { 2 - 4 } \multicolumn{1}{c|}{} & Hombre & Mujer & Total \\
\hline \multirow{2}{*}{ Así } & 381 & 549 & 930 \\
\cline { 2 - 4 } & $87,4 \%$ & $94,0 \%$ & $90 \%$ \\
\hline \multirow{2}{*}{ Asín } & 55 & 35 & 90 \\
\cline { 2 - 4 } & $12,6 \%$ & $6,0 \%$ & $8,8 \%$ \\
\hline \multirow{2}{*}{ Total } & 436 & 584 & 1020 \\
\cline { 2 - 4 } & $100 \%$ & $100 \%$ & $100 \%$ \\
\hline \multirow{2}{*}{ Coeficiente Chi-cuadrado de Pearson: $13,605(1)$. Sig.: $>, 001$} \\
V de Cramer: 0,115. Sig.: $>, 001$ \\
Phi: -0,115. Sig.: >,001
\end{tabular}

Tabla 16. Realizaciones de así según el sexo. Frecuencias absolutas, relativas y significación estadística.

Respecto a la influencia de la edad en la realización, se obtuvieron los siguientes datos:

\begin{tabular}{|c|c|c|c|c|}
\cline { 2 - 5 } \multicolumn{1}{c|}{} & $20-34$ años & $35-54$ años & +55 años & Total \\
\hline \multirow{3}{*}{ Así } & 389 & 242 & 299 & 930 \\
\cline { 2 - 5 } & $94,2 \%$ & $90,6 \%$ & $87,9 \%$ & $91,2 \%$ \\
\hline \multirow{2}{*}{ Asín } & 24 & 25 & 41 & 90 \\
\cline { 2 - 5 } & $5,8 \%$ & $9,4 \%$ & $12,1 \%$ & $8,8 \%$ \\
\hline \multirow{2}{*}{ Total } & 413 & 267 & 340 & 1020 \\
\cline { 2 - 5 } & $100 \%$ & $100 \%$ & $100 \%$ & $100 \%$ \\
\hline \multirow{2}{*}{ Coeficiente Chi-cuadrado de Pearson: 9,179 (2). Sig.: ,010 } \\
V de Cramer: 0,095. Sig.: ,010 \\
Phi: 0,095. Sig.: ,010 \\
\hline
\end{tabular}

Tabla 17. Realizaciones de así según la edad. Frecuencias absolutas, relativas y significación estadística.

Se puede observar que la generación más joven es la que favorece la realización de así (94,2 \%). Por lo tanto, los mayores presentan un mayor índice de asín (12,1\%). Aunque la significación es menor en este caso, la relación entre las variables sigue siendo significativa. 


\begin{tabular}{|c|c|c|c|c|}
\cline { 2 - 5 } \multicolumn{1}{c|}{} & $\begin{array}{c}\text { Sin estudios } \\
\text { Estudios primarios }\end{array}$ & Secundaria & $\begin{array}{c}\text { Estudios } \\
\text { superiores }\end{array}$ & Total \\
\hline \multirow{3}{*}{ Así } & 192 & 352 & 386 & 930 \\
\cline { 2 - 5 } & $86,9 \%$ & $89,8 \%$ & $94,8 \%$ & $91,2 \%$ \\
\hline \multirow{3}{*}{ Asín } & 29 & 40 & 21 & 90 \\
\cline { 2 - 5 } & $13,1 \%$ & $10,2 \%$ & $5,2 \%$ & $8,8 \%$ \\
\hline \multirow{2}{*}{ Total } & 221 & 392 & 407 & 1020 \\
\cline { 2 - 5 } & $100 \%$ & $100 \%$ & $100 \%$ & $100 \%$ \\
\hline \multirow{2}{*}{ Coeficiente Chi-cuadrado de Pearson: 12,796 (2). Sig.: , 002} \\
V de Cramer: 0,112. Sig.: , 005 \\
Phi: 0,112. Sig.: ,005 \\
\hline
\end{tabular}

Tabla 18. Realizaciones de así según el nivel educativo. Frecuencias absolutas, relativas y significación estadística.

Por último, la tabla 18, recoge la influencia del nivel educativo en la realización de esta variable. Se observa que los informantes con estudios superiores realizan en un $94,8 \%$ de las ocasiones así. La forma asín es, por tanto, propia de hombres mayores con niveles educativos bajos.

\subsection{Vulgarismos como resultado de procesos de debilitamiento de las consonantes o de una monoptongación}

\subsubsection{Pues}

La forma pues alterna con las realizaciones pue, pos y po. Las variantes fueron recodificadas según la clasificación presentada en la tabla 19. La poca influencia del segmento /-s/ en la elección de una u otra variable parece tener relación con el fenómeno que ocurre en Málaga, estudiado por Vida (2004), mediante el cual se tiende a elidir este segmento en posición final de palabra.

\begin{tabular}{|c|c|c|}
\cline { 2 - 3 } \multicolumn{1}{c|}{} & Frecuencia & Porcentaje \\
\hline $\begin{array}{c}\text { Pues } \\
\text { Pue }\end{array}$ & 3987 & $90,94 \%$ \\
\hline $\begin{array}{c}\text { Pos } \\
\text { Po }\end{array}$ & 397 & $9,05 \%$ \\
\hline Total & 4384 & $100 \%$ \\
\hline
\end{tabular}

Tabla 19. Frecuencias absolutas y relativas de las realizaciones de pues.

En cuanto a la influencia del sexo de los hablantes en la realización de la variable, se obtuvieron los datos recogidos en la tabla 20. 


\begin{tabular}{|c|c|c|c|}
\hline & Hombre & Mujer & Total \\
\hline \multirow{2}{*}{$\begin{array}{c}\text { Pues } \\
\text { Pue }\end{array}$} & 1921 & 2066 & 3987 \\
\hline & $89,4 \%$ & $92,4 \%$ & $90,0 \%$ \\
\hline \multirow{2}{*}{$\begin{array}{c}\text { Pos } \\
\text { Po }\end{array}$} & 227 & 170 & 397 \\
\hline & $10,6 \%$ & $7,6 \%$ & $9,1 \%$ \\
\hline \multirow{2}{*}{ Total } & 2148 & 2236 & 4384 \\
\hline & $100 \%$ & $100 \%$ & $100 \%$ \\
\hline \multicolumn{4}{|c|}{$\begin{array}{l}\text { Coeficiente Chi-cuadrado de Pearson: 11,696 (1). Sig.: ,001 } \\
\text { V de Cramer: 0,052. Sig.: ,001 } \\
\text { Phi: -0,052. Sig.: ,001 }\end{array}$} \\
\hline
\end{tabular}

Tabla 20. Realizaciones de pues según el sexo. Frecuencias absolutas, relativas y significación estadística.

Los hombres presentan un porcentaje levemente mayor de realizaciones de pos y po $(10,6 \%)$, frente a las mujeres que se muestran más conservadoras $(7,6 \%)$.

En cuanto al nivel educativo, se optó por dividir la muestra de informantes en dos grupos ya que se observó que los hablantes sin estudios mostraban un patrón contrario a los que habían recibido algún tipo de educación.

\begin{tabular}{|c|c|c|c|}
\cline { 2 - 4 } \multicolumn{1}{c|}{} & Sin estudios & Con estudios & Total \\
\hline \multirow{2}{*}{$\begin{array}{c}\text { Pues } \\
\text { Pue }\end{array}$} & 261 & 3726 & 3987 \\
\cline { 2 - 4 } $\begin{array}{c}\text { Pos } \\
\text { Po }\end{array}$ & $73,3 \%$ & $92,5 \%$ & $90,9 \%$ \\
\hline \multirow{2}{*}{ Total } & 95 & 302 & 397 \\
\cline { 2 - 4 } & $26,7 \%$ & $7,5 \%$ & $43 \%$ \\
\hline \multicolumn{3}{|c|}{$\begin{array}{c}\text { Coeficiente Chi-cuadrado de Pearson: } 146,227 \text { (1). Sig.: }>, 001 \\
\text { V de Cramer: 0,183. Sig.: }>, 001 \\
\text { Phi: -0,183. Sig.: }>, 001\end{array}$} \\
\hline
\end{tabular}

Tabla 21. Realizaciones de pues según el nivel educativo. Frecuencias absolutas, relativas y significación estadística.

La tabla 21 muestra diferencias significativas en cuanto a la incidencia del nivel educativo en la realización de la variable pues. Se podría concluir que la reducción del diptongo está favorecida por los hombres sin estudios. Cabe mencionar que, aunque se analizó el factor edad, su influencia en esta variable no fue significativa.

\subsubsection{Muy}

La forma muy se realiza a menudo como $m u$. En la tabla 22 se comprueba que esta última realización alcanza un 32,7\% de los casos, si bien la variante estándar sigue siendo mayoritaria. 


\begin{tabular}{|c|c|c|}
\cline { 2 - 3 } \multicolumn{1}{c|}{} & Frecuencia & Porcentaje \\
\hline Muy & 1770 & $67,3 \%$ \\
\hline $\mathrm{Mu}$ & 859 & $32,7 \%$ \\
\hline Total & 2629 & $100 \%$ \\
\hline
\end{tabular}

Tabla 22. Frecuencias absolutas y relativas de las realizaciones de muy

\begin{tabular}{|c|c|c|c|}
\cline { 2 - 4 } \multicolumn{1}{c|}{} & Hombre & Mujer & Total \\
\hline \multirow{3}{*}{ Muy } & 806 & 964 & 1770 \\
\cline { 2 - 4 } & $71,3 \%$ & $64,3 \%$ & $67,3 \%$ \\
\hline \multirow{2}{*}{ Mu } & 324 & 535 & 859 \\
\cline { 2 - 4 } & $28,7 \%$ & $35,7 \%$ & $32,7 \%$ \\
\hline \multirow{2}{*}{ Total } & 1130 & 1499 & 2629 \\
\cline { 2 - 4 } & $100 \%$ & $100 \%$ & $100 \%$ \\
\hline \multirow{2}{*}{ Coeficiente Chi-cuadrado de Pearson: $14,425(1)$. Sig.: $>, 001$} \\
V de Cramer: 0,074. Sig.: $>, 001$ \\
Phi: 0,074. Sig.: $>, 001$
\end{tabular}

Tabla 23. Realizaciones de muy según el sexo. Frecuencias absolutas, relativas y significación estadística

En la tabla 23 se observa que son las mujeres las que favorecen la aparición de $m u$ $(35,7 \%)$, estableciéndose una relación estadísticamente significativa.

\begin{tabular}{|c|c|c|c|c|}
\cline { 2 - 5 } \multicolumn{1}{c|}{} & $20-34$ años & $35-54$ años & +55 años & Total \\
\hline \multirow{3}{*}{ Muy } & 560 & 597 & 613 & 1770 \\
\cline { 2 - 5 } & $56,2 \%$ & $71,4 \%$ & $77,0 \%$ & $67,3 \%$ \\
\hline \multirow{3}{*}{ Mu } & 437 & 239 & 183 & 859 \\
\cline { 2 - 5 } & $43,8 \%$ & $28,6 \%$ & $23,0 \%$ & $32,7 \%$ \\
\hline \multirow{3}{*}{ Total } & 997 & 836 & 796 & 2629 \\
\cline { 2 - 5 } & $100 \%$ & $100 \%$ & $100 \%$ & $100 \%$ \\
\hline \multirow{2}{*}{ Coeficiente Chi-cuadrado de Pearson: $96,699(2)$. Sig.: $>, 001$} \\
V de Cramer: 0,192. Sig.: $>, 001$ \\
Phi: 0,192. Sig.: >,001 \\
\hline
\end{tabular}

Tabla 24. Realizaciones de muy según la edad. Frecuencias absolutas, relativas y significación estadística. 
Teniendo en cuenta la edad (tabla 24), la generación más joven es la que favorece la realización $m u(43,8 \%)$, ya que el porcentaje de ocurrencias va disminuyendo a medida que aumenta la edad del informante.

\begin{tabular}{|c|c|c|c|c|}
\cline { 2 - 5 } \multicolumn{1}{c|}{} & $\begin{array}{c}\text { Sin estudios } \\
\text { Estudios primarios }\end{array}$ & Secundaria & $\begin{array}{c}\text { Estudios } \\
\text { superiores }\end{array}$ & Total \\
\hline \multirow{3}{*}{ Muy } & 167 & 806 & 797 & 1770 \\
\cline { 2 - 5 } & $40,6 \%$ & $72,1 \%$ & $72,5 \%$ & $67,3 \%$ \\
\hline \multirow{2}{*}{ Mu } & 244 & 312 & 303 & 90 \\
\cline { 2 - 5 } & $59,4 \%$ & $27,9 \%$ & $27,5 \%$ & $32,7 \%$ \\
\hline \multirow{2}{*}{ Total } & 411 & 1118 & 1100 & 2629 \\
\cline { 2 - 5 } & $100 \%$ & $100 \%$ & $100 \%$ & $100 \%$ \\
\hline \multirow{2}{*}{ Coeficiente Chi-cuadrado de Pearson: $157,828(2)$. Sig.: $>, 001$} \\
V de Cramer: 0,245. Sig.: $>, 001$ \\
Phi: 0,245. Sig.: >,001
\end{tabular}

Tabla 25. Realizaciones de muy según el nivel educativo.

Frecuencias absolutas, relativas y significación estadística.

Al estudiar el efecto del nivel educativo en la variación de muy, se observa que son los hablantes menos instruidos los que favorecen el uso de la forma reducida $m u(59,4 \%)$.

Lo más llamativo de esta distribución, es que la forma $m u$ sea propia de mujeres jóvenes, lo que indica un comportamiento diferente al que ofrece el resto de vulgarismos.

\subsubsection{Nada}

La forma nada (tabla 26) es mayoritaria en los materiales analizados (65,9\%), frente a su variante, la forma $n a(34,1 \%)$.

\begin{tabular}{|c|c|c|}
\cline { 2 - 3 } \multicolumn{1}{c|}{} & Frecuencia & Porcentaje \\
\hline Nada & 931 & $65,9 \%$ \\
\hline $\mathrm{Na}$ & 482 & $34,1 \%$ \\
\hline Total & 1413 & $100 \%$ \\
\hline
\end{tabular}

Tabla 26. Frecuencias absolutas y relativas de las realizaciones de nada.

En la tabla 27 se observa que son los hombres los que favorecen la realización de la variante reducida $(39,9 \%)$. Las diferencias entre los grupos son estadísticamente significativas. 


\begin{tabular}{|c|c|c|c|}
\hline & Hombre & Mujer & Total \\
\hline \multirow{2}{*}{ Nada } & 365 & 566 & 931 \\
\hline & $60,1 \%$ & $70,2 \%$ & $65,9 \%$ \\
\hline \multirow{2}{*}{$\mathrm{Na}$} & 242 & 240 & 482 \\
\hline & $39,9 \%$ & $29,8 \%$ & $34,1 \%$ \\
\hline \multirow{2}{*}{ Total } & 607 & 806 & 1413 \\
\hline & $100 \%$ & $100 \%$ & $100 \%$ \\
\hline \multicolumn{4}{|c|}{$\begin{array}{c}\text { Coeficiente Chi-cuadrado de Pearson: } 15,689 \text { (1). Sig.: >,001 } \\
\text { V de Cramer: 0,105. Sig.: >,001 } \\
\text { Phi: }-0,105 \text {. Sig.: }>, 001\end{array}$} \\
\hline
\end{tabular}

Tabla 27. Realizaciones de nada según el sexo. Frecuencias absolutas, relativas y significación estadística.

\begin{tabular}{|c|c|c|c|}
\hline & 20-34 años & +35 años & Total \\
\hline \multirow{2}{*}{ Nada } & 329 & 602 & 931 \\
\hline & $57,7 \%$ & $71,4 \%$ & $65,9 \%$ \\
\hline \multirow{2}{*}{$\mathrm{Na}$} & 241 & 241 & 482 \\
\hline & $42,3 \%$ & $28,6 \%$ & $34,1 \%$ \\
\hline \multirow{2}{*}{ Total } & 570 & 843 & 1413 \\
\hline & $100 \%$ & $100 \%$ & $100 \%$ \\
\hline \multicolumn{4}{|c|}{$\begin{array}{c}\text { Coeficiente Chi-cuadrado de Pearson: 28,366 (1). Sig.: >,001 } \\
\text { V de Cramer: 0,142. Sig.: }>, 001 \\
\text { Phi: }-0,142 \text {. Sig.: }>, 001\end{array}$} \\
\hline
\end{tabular}

Tabla 28. Realizaciones de nada según la edad. Frecuencias absolutas, relativas y significación estadística.

En cuanto a la edad, se observa que el grupo generacional de los hablantes más jóvenes son los que favorecen la realización de na con un 42,3\%.

Con respecto al nivel educativo, se observa que son los hablantes menos instruidos los que promocionan la variante reducida $n a(54,7 \%)$. La frecuencia de aparición de esta variable va reduciéndose a medida que se asciende en la escala educacional.

Al igual que ocurre en el caso anterior, la significación obtenida es máxima. Por lo tanto, se puede afirmar la existencia de una relación entre las variables.

La elección de la forma na parece ser propia de hombres jóvenes con bajo nivel de instrucción. 


\begin{tabular}{|c|c|c|c|c|}
\cline { 2 - 5 } \multicolumn{1}{c|}{} & $\begin{array}{c}\text { Sin estudios } \\
\text { Estudios primarios }\end{array}$ & Secundaria & $\begin{array}{c}\text { Estudios } \\
\text { superiores }\end{array}$ & Total \\
\hline \multirow{3}{*}{ Nada } & 182 & 391 & 358 & 931 \\
\cline { 2 - 5 } & $45,3 \%$ & $72,5 \%$ & $75,8 \%$ & $65,9 \%$ \\
\hline \multirow{2}{*}{$\mathrm{Na}$} & 220 & 148 & 114 & 482 \\
\cline { 2 - 5 } & $54,7 \%$ & $27,5 \%$ & $24,2 \%$ & $34,1 \%$ \\
\hline \multirow{2}{*}{ Total } & 402 & 539 & 472 & 1413 \\
\cline { 2 - 5 } & $100 \%$ & $100 \%$ & $100 \%$ & $100 \%$ \\
\hline \multirow{2}{*}{ Coeficiente Chi-cuadrado de Pearson: $107,455(2)$. Sig.: $>, 001$} \\
V de Cramer: 0,276. Sig.: $>, 001$ \\
Phi: 0,276. Sig.: >,001 \\
\hline
\end{tabular}

Tabla 29. Realizaciones de nada según el nivel educativo.

Frecuencias absolutas, relativas y significación estadística.

\subsubsection{Para}

En la tabla 30 se observa la alternancia de las formas para y pa. Se observa que las frecuencias de ambas formas están relativamente equilibradas, si bien la forma reducida $p a$ es algo más frecuente $(56,0 \%)$.

\begin{tabular}{|c|c|c|}
\cline { 2 - 3 } \multicolumn{1}{c|}{} & Frecuencia & Porcentaje \\
\hline Para & 1172 & $44,0 \%$ \\
\hline $\mathrm{Pa}$ & 1429 & $56,0 \%$ \\
\hline Total & 2664 & $100 \%$ \\
\hline
\end{tabular}

Tabla 30. Frecuencias absolutas y relativas de las realizaciones de para.

Con respecto a la edad (tabla 31 ) se puede afirmar que la variante más frecuente $(\mathrm{pa})$ se ve favorecida por los jóvenes $(61,6 \%$ ) y su frecuencia va disminuyendo a medida que avanzamos en la escala.

Los datos obtenidos de las pruebas estadísticas ponen de manifiesto la relación existente entre las variables.

Si se atiende al nivel educativo de los hablantes (tabla 32), se observa que, en el caso de los informantes sin estudios, predomina el uso de $p a(90,1 \%)$. La diferencia se hace menos marcada a medida que se avanza en la escala.

Por último, se estudió la influencia del tema de conversación. Este aspecto se analizó con más detalle en esta variable ya que las realizaciones de para y pa ofrecieron una ocurrencia muy similar. Para ello se clasificaron los temas según si trataban asuntos cotidianos o si abordaban temas más serios (mayor implicación personal). 


\begin{tabular}{|c|c|c|c|c|}
\cline { 2 - 5 } \multicolumn{1}{c|}{} & $20-34$ años & $35-54$ años & +55 años & Total \\
\hline \multirow{2}{*}{$\begin{array}{c}\text { Para } \\
38,4 \%\end{array}$} & 347 & 363 & 462 & 1172 \\
\cline { 2 - 5 } & $44,2 \%$ & $49,1 \%$ & $44,0 \%$ & 1492 \\
\hline \multirow{2}{*}{$\begin{array}{c}\text { Pa } \\
61,6 \%\end{array}$} & 556 & 458 & 478 & 2664 \\
\hline \multirow{2}{*}{ Total } & $55,8 \%$ & $50,9 \%$ & $56,0 \%$ & 940 \\
\hline $100 \%$ & 903 & 821 & $100 \%$ & \\
\cline { 2 - 5 } & $100 \%$ & $100 \%$ & Coeficiente Chi-cuadrado de Pearson: $21,510(2)$. Sig.: $>, 001$ \\
V de Cramer: 0,090. Sig.: $>, 001$ \\
Phi: 0,090. Sig.: $>, 001$
\end{tabular}

Tabla 31. Realizaciones de para según la edad.

Frecuencias absolutas, relativas y significación estadística.

\begin{tabular}{|c|c|c|c|c|c|}
\cline { 2 - 6 } \multicolumn{1}{c|}{} & Sin estudios & Estudios primarios & Secundaria & $\begin{array}{c}\text { Estudios } \\
\text { superiores }\end{array}$ & Total \\
\hline \multirow{2}{*}{ Para } & 29 & 129 & 492 & 522 & 1172 \\
\cline { 2 - 6 } & $9,9 \%$ & $39,8 \%$ & $44,8 \%$ & $55,1 \%$ & $44,0 \%$ \\
\hline \multirow{2}{*}{$\mathrm{Pa}$} & 265 & 195 & 606 & 426 & $90,1 \%$ \\
\cline { 2 - 6 } & $90,1 \%$ & $60,2 \%$ & $55,1 \%$ & $44,9 \%$ & $56,0 \%$ \\
\hline \multirow{2}{*}{ Total } & 294 & 324 & 1098 & 948 & 2664 \\
\cline { 2 - 6 } & $100 \%$ & $100 \%$ & $100 \%$ & $100 \%$ & $100 \%$ \\
\hline \multicolumn{6}{|c|}{ Coeficiente Chi-cuadrado de Pearson: $188,729(3)$. Sig.: $>, 001$} \\
V de Cramer: 0,266. Sig.: $>, 001$ \\
Phi: 0,266. Sig.: >,001 \\
\cline { 2 - 5 }
\end{tabular}

Tabla 32. Realizaciones de para según el nivel educativo.

Frecuencias absolutas, relativas y significación estadística.

\begin{tabular}{|c|c|c|c|}
\cline { 2 - 4 } \multicolumn{1}{c|}{} & $\begin{array}{c}\text { Biografía y familia } \\
\text { Barrio y cultura local }\end{array}$ & Temas «serios» & Total \\
\hline \multirow{2}{*}{ Para } & 1108 & 64 & 1172 \\
\cline { 2 - 4 } & $43,2 \%$ & $66,0 \%$ & $44,0 \%$ \\
\hline \multirow{2}{*}{$\mathrm{Pa}$} & 1459 & 33 & 1492 \\
\cline { 2 - 4 } & $56,8 \%$ & $34,0 \%$ & $56,0 \%$ \\
\hline \multirow{2}{*}{ Total } & 2567 & 97 & $100 \%$ \\
\cline { 2 - 4 } & $100 \%$ & $100 \%$ & 1064 \\
\hline \multirow{2}{*}{$\begin{array}{r}\text { Coeficiente Chi-cuadrado de Pearson: } 19,748(1) . \text { Sig.: }>, 001 \\
\text { V de Cramer: 0,086. Sig.: }>, 001 \\
\text { Phi: }-0,086 . \text { Sig.: }>, 001\end{array}$} \\
\cline { 2 - 2 }
\end{tabular}

Tabla 33. Realizaciones de para según el tema.

Frecuencias absolutas, relativas y significación estadística. 
En la tabla 33 se observa que la frecuencia de uso de pa es mayor en los contextos más informales $(56,8 \%$, frente al $34 \%$ de los temas más serios).

En esta variable, el estudio de la incidencia del sexo en la variable demostró que no presentaba una influencia representativa.

\section{INTERPRETACIÓN DE LOS RESULTADOS}

\subsection{Segmento fonológico /-n/}

Los resultados obtenidos muestran que en Málaga se impone de manera casi categórica la velarización, esto se puede observar en el 95,4 \% que presenta esta variante (tabla 5). Esta superioridad se mantiene en todos los contextos. La realización velar en estudios anteriores predomina en el contexto prepausal. Esta afirmación se puede comprobar en la tabla 1, donde los valores de la articulación velar ante pausa son de 69,3\% en el caso de Puerto Rico (López Morales, 1983: 109), de 83 \% en Miami (Hammond, 1979: 19-36) y de 79,82 \% en Las Palmas (Samper Padila, 1990: 221). Esto contrasta con los resultados obtenidos por Terrell en La Habana (1975: 257-271), donde, salvo en el contexto prepausal, la realización velar no resulta significativa. En Filadelfia (Poplack, 1979: 129-143), la realización velar ante consonante también contrasta con la de este estudio, ya que se observa un valor del $5 \%$, frente al 95,9 \% de Málaga. Lo mismo ocurre con el estudio de Samper Padilla (1990: 229-238) en Las Palmas, donde ante consonante la velarización ocurre en un 9,89 \%. En Las Palmas la velar solo es promocionada por el contexto prepausal. Se observa que el contexto prepausal en Málaga es el que favorece levemente la elisión de la nasal. Esto también ocurre en Puerto Rico (López Morales, 1983: 109), donde se observa un 8,1 \% comparable con el $8,8 \%$ recogido en la ciudad de Málaga. Las realizaciones alveolares y asimiladas carecen de significación en Málaga, mientras que en otros estudios ocurre lo contrario. En el caso de Puerto Rico (López Morales, 1983: 109) se recoge un 80,6 \% ante consonante. Lo mismo ocurre en Las Palmas (Samper Padilla, 1990: 221), donde se observa un 82,17 \% y un 54,23\% ante vocal. Sin embargo, el estudio de Panamá (Cedergren, 1973) muestra la misma tendencia que Málaga.

Otro rasgo destacable es que se muestra una leve tendencia a elidir la nasal $(5,4 \%)$ cuando cumple una función morfológica. Esto también sucede en Las Palmas (Samper Padilla 1990: 229-238) y en Filadelfia (Poplack 1979: 129-143), como se mencionó anteriormente.

Los resultados en cuanto al análisis de la distribución generacional indican que son los informantes de más de 55 años los que favorecen su elisión (4,8 \%). A la misma conclusión llego Samper Padilla (1990: 255) en su estudio sobre Las Palmas. Sin embargo, en el trabajo de López Morales (1983: 109-110) en Puerto Rico, la edad no resulta un factor significativo.

En cuanto al nivel educacional, en Málaga son los estratos socioculturales más bajos los que propician levemente la elisión (3,8 \%). Lo mismo afirmaron Samper Padilla (1990: 255) y López Morales (1983: 109-110).

Por último, se analizó la influencia del sexo de los hablantes, se observa que son los hombres quienes promocionan la elisión (4,1 \%). Lo contrario ocurre en Las Palmas (Samper Padilla, 1990: 255), donde son las mujeres quienes promocionan el cero fonético.

Se podría afirmar que, en cuanto al contexto lingüístico, la velarización se impone de manera casi categórica ante todos los contextos. No obstante, el contexto prepausal y la 
transmisión de un valor morfológico propician la elisión. En cuanto a los factores sociales, es la generación de mayor edad, junto con los hombres y los niveles socioculturales más bajos los que favorecen la aparición del cero fonético.

Para obtener una visión general del mundo hispánico, aunque no se observan tendencias estrictamente homogéneas, se puede afirmar, que el español de América presenta una tendencia similar a la observada en Málaga. En estas dos zonas geográficas se observan elevados porcentajes de velarización y elisión (a excepción de Puerto Rico según el estudio de López Morales en 1983: 107), por lo que se trataría de zonas con dialectos innovadores. Sin embargo, la situación de Las Palmas (Samper Padilla, 1990: 217) difiere de las anteriores y se asimila a la encontrada en Puerto Rico (López Morales, 1983: 107). En este caso se observa una mayor frecuencia de realizaciones asimiladas y alveolares que velarizaciones y elisiones. No obstante, la velarización cobra importancia ante el contexto pausal, lo que podría indicar el inicio del proceso de velarización.

Otro hecho destacable es que en Málaga la realización velar se impone como categórica, mientras este fenómeno no ocurre en el resto de ciudades estudiadas.

\subsection{Vulgarismos}

Con respecto al uso de los vulgarismos analizados, se puede decir que se conserva la realización «normativa» en casi todos los casos: luego (97,9\%), pues $(90,94 \%)$, asi $(91,2 \%)$, muy $(67,3 \%)$ y nada $(65,9 \%)$. No obstante, la variable para es la única que ofrece un porcentaje relativamente alto de la variante debilitada (56\%). Esto puede ser consecuencia del proceso evolutivo de las consonantes (Penny, 2014: 57-106). La dental, tal y como observan Blanco (2004: 122-137) y Villena y Moya (2016: 281-321), tiende a perderse en el español peninsular. Asimismo, cabe recordar que Moya y García (2009: 92-123) afirmaron que el debilitamiento y pérdida de la dental sonora llega a afectar a unidades léxicas de uso frecuente, como sería el caso de nada. Por otro lado, el hecho de que la 'o' breve tónica del latín diptongase en 'ue' en español puede indicar un retroceso en la historia de la palabra. En cuanto a la variable $a s i$, se puede observar una tendencia similar a la recogida en el AleCman (García y Moreno, 2003).

Como tendencias generales se puede extraer:

1. Aluego y asín siguen un patrón similar al ser realizaciones propias de hombres mayores y con niveles educacionales bajos.

2. Pos y po se comportan de forma parecida a las anteriores, al estar favorecidas por hombres con bajo nivel de instrucción, aunque su presencia es relativamente estable en los diferentes grupos generacionales.

3. $М u$ у na parecen seguir otra dirección. Aunque no son realizaciones mayoritarias, son relativamente frecuentes y están promocionadas por los hablantes más jóvenes con bajo nivel de instrucción. Como sucedía anteriormente, los hombres favorecen las realizaciones alternativas en el caso de $n a$. Sin embargo, en el caso de mu son las mujeres quienes propician la variante debilitada.

4. Por último, la forma $p a$ sí es mayoritaria frente a la variante estándar para y también está favorecida por los hombres jóvenes de nivel educacional bajo. 
En resumen, se podría afirmar que en Málaga, aunque predominan las realizaciones normativas, las realizaciones «vulgares» están favorecidas por los hombres con bajo nivel de instrucción. Por otra parte, se aprecian dos patrones diferenciados que parecen relacionarse con el proceso fonético implicado: los vulgarismos procedentes de los refuerzos fonéticos (asín y aluego) son propios de los hablantes de mayor edad, por lo que se puede intuir que están en retroceso. Sin embargo, las formas que surgen a partir de los procesos de debilitamiento de las consonantes intervocálicas ( $n a$ y $p a$ ) o de una monoptongación ( $p o$ y $m u$ ), son más frecuentes entre las generaciones más jóvenes, que las promocionan.

El comportamiento de los hablantes nos permite suponer que estas formas ( $p a, n a, m u$, etc.) son propias del estilo coloquial. Algunas, sobre todo aluego y asín, parecen estar estigmatizadas, por lo que su realización se identifica con los estratos socioeconómicos y los niveles culturales más bajos. Por esto se podría decir que representan formas «vulgares» del español.

\section{CONCLUSIÓN}

A partir de los resultados presentados, se pueden extraer las siguientes conclusiones:

1. En Málaga, la variante más frecuente de /-n/ en final de palabra es la velar, que se impone de manera casi categórica. En cuanto a la elisión del segmento implosivo, son los hombres mayores con estudios bajos los que la favorecen esta tendencia.

2. Con respecto a las formas vulgares, se observan los siguientes patrones:

a. Luego. En el caso de la variante aluego, se observa que su realización está favorecida por los hombres, mayores y con estudios bajos, lo que destacaría un carácter aparentemente vulgar, según las afirmaciones de López Morales (2004: 56-180) y Moreno Fernández (2009: 177-190).

b. Así. También son los hombres mayores con bajo nivel educativo quienes favorecen la forma asin.

c. Pues. Una vez más, son los hombres sin estudios los que promocionan la reducción del diptongo. En este caso no se observa una influencia clara de la edad en la alternancia de variantes.

d. Muy. Esta variante, a diferencia de las anteriores, está promocionada por las mujeres jóvenes sin estudios.

e. Nada. Tal y como se observa en el trabajo de Moya et alii (2012: 95-148) en la ciudad de Granada, la presencia de na se ve favorecida por hablantes jóvenes de niveles educacionales bajos. No obstante, en el caso de Málaga, se observa que los hombres tienden más a la elisión que las mujeres, mientras que en Granada el sexo no resulta significativo.

f. Para. En este caso, la variante debilitada pa es más frecuente que la estándar y está promocionada por los jóvenes sin estudios.

Se observa, por tanto, que las formas aluego, asin y pos son favorecidas por las personas de mayor edad y que, probablemente, tenderán a desaparecer. Por otro lado, las formas $p a$, na y $m u$ son propias de hablantes jóvenes con niveles de instrucción bajos, lo que lleva a pensar que son variantes que gozan de cierta vitalidad, especialmente la forma debilitada $p a$, que es más frecuente que la normativa para. 
Por otro lado, la variable para parece estar condicionada por el estilo, ya que la presencia del coloquialismo pa es más frecuente cuando el tema de conversación es más informal.

En cuanto a la nasal implosiva, tras establecer la comparación entre los datos obtenidos para el segmento /-n/ en final de palabra en Málaga y los obtenidos en el resto del mundo hispánico, se puede afirmar que el proceso de debilitamiento de la nasal se encuentra estable en un punto intermedio de velarización. Esta solución es, por otra parte, bastante frecuente en la mayor parte de las zonas investigadas, la única excepción es el caso de Panamá (Cedergren, 1973), donde el cero fonético presenta porcentajes muy elevados, por lo que se puede considerar que el proceso se encuentra en un estado más avanzado.

Por otro lado, la elisión del segmento es más frecuente cuando presenta función morfemática. Esta tendencia también aparece recogida en estudios anteriores como el de Samper Padilla (1990: 229-238) y el de Poplack (1979: 129-143), así como en estudios realizados sobre el segmento /-s/ (Vida, 2004: 132-139), según los cuales, la lengua tiende a eliminar segmentos reiterativos.

En resumen, se puede concluir que en la variedad innovadora de Málaga el proceso de debilitamiento de /-n/ implosiva no está tan avanzado como el de /-s/ final, ya que la variante más frecuente en posición final de palabra es la velar. Por otro lado, la elisión del segmento está favorecida por hablantes mayores con bajo nivel de instrucción. Este comportamiento se asemeja al que presentan las formas léxicas que se refuerzan fonéticamente: luego y asi (aluego y asín). Sin embargo, las formas que presentan debilitamiento fonético están más extendidas entre la población y son propias del habla rápida y coloquial.

\section{Referencias bibliográficas}

Alvar, M. (1966). «El español de Tenerife. Cuestión de principios», Zeitschrift für Romanische Philologie, LXXXII, pp. 507-548.

Alvar, M. (1972). Niveles socio-culturales en el habla de Las Palmas de Gran Canaria. Las Palmas de Gran Canaria: Ed. del Excmo. Cabildo Insular de Gran Canaria.

Blanco Canales, A. (2004). Estudio sociolingüistico de Alcalá de Henares. Alcalá de Henares: Servicio de publicaciones de la Universidad de Alcalá.

Bourdieu, P. (1977). «L'économie des échanges linguistiques», $L F r$, 34, pp.17-34

Cedergren, H. (1973). Interplay of social and linguistic factors in Panama (tesis doctoral inédita). Ithaca: Cornell University.

Cedegren, H. (1983). «Sociolingüística». En López Morales, H. (ed.) Estratificación social del español de San Juan de Puerto Rico. México, D.F.: Universidad Nacional Autónoma de México, pp. 147-165.

Fasold, R. (1996). «La estadística», en La sociolingüistica de la sociedad. Introducción a la sociolingüistica. Madrid: Visor.

García Mouton, P. y F. Moreno Fernández. (2003). Atlas Lingüistico (y etnográfico) de Castilla - La Mancha. Universidad de Alcalá http://www2.uah.es/alecman (01-10-2017)

Haché de Yunén, A. M. (1982). «La/n/ final de sílaba en el español de Santiago de los Caballeros». En Alba, O. (ed.), El español del Caribe. Santiago de los Caballeros: Universidad Católica Madre y Maestra, pp. 145-154.

Hammond, R. (1979). «The velar nasal in rapid Cuban Spanish». En J. P. Lantolf, F. H. W. Frank, J. M. Guitart (eds.), Colloquium on Spanish and Luso-Brazilian Linguistics, Washington D.C.: Georgetown University Press, pp. 19-36.

Hauser, R. (2012). Velarización de /n/y nasalización vocálica en las hablas de Sevilla y Granada (plan de trabajo de la tesis de licenciatura). Lizentiatsarbeit Universität Zürich: Zürich. 
López Morales, H. (1980). «Sociolingüística hispánica: perspectivas futuras», Perspectivas de la investigación lingüistica en Hispanoamérica. México: Universidad Nacional Autónoma de México, pp. 59-78.

López Morales, H. (1983). Estratificación social del español de San Juan de Puerto Rico. México, D.F.: Universidad Nacional Autónoma de México.

López Morales, H. (2004). Sociolingüística $3^{\mathrm{a}}$ ed. Madrid: Gredos, pp. 56-180.

Lorenzo, A. (1976). El habla de Los Silos. Santa Cruz de Tenerife: Caja general de Ahorros de Santa Cruz de Tenerife.

Ma, R., Herasimchuk, E. (1972). «The linguistic dimensions of a bilingual neighborhood». En Fishman, J. et al. (eds.), Bilingualism in the barrio, Bloomington: Indiana University Press, pp. 349-464.

Moreno Fernández, F. (1996). «Metodología del Proyecto para el Estudio Sociolingüístico del Español de España y de América (PRESEEA)», Lingüística, 8, pp. 257-287.

Moreno Fernández, F. (2009). Principios de sociolingüistica y sociología del lenguaje $4^{\mathrm{a}}$ ed. Barcelona: Ariel.

Moya Corral, J. A. y E. J. García Wiedemann. (2009). «La elisión de /d/ intervocálica en el español culto de Granada: factores lingüísticos», Pragmalingüística, 17, pp. 92-123

Moya Corral, J. A., E. J. García Wiedemann, E. T. Montoro del Arco, M. Sosinski, M. C. Torres López, F. Pose Furest, E. Melguizo Moreno. (2012). «La /d/ intervocálica en Granada: factores lingüísticos y sociales». En E. Waluch de la Torre y J. A. Moya Corral (coord.), Español hablado. Estudios sobre el corpus PRESEEA-Granada. Varsovia: Universidad de Varsovia, pp. 95-148.

Narbona, A., Cano, R. y Morillo, R. (2003): El español hablado en Andalucía. Sevilla: Fundación José Manuel Lara.

Penny, R. (2014). Gramática histórica del español. Barcelona: Ariel.

Poplack, S. (1979). «Sobre la elisión y la ambigüedad en el español puertorriqueño: el caso de la /n\#/ verbal», Boletín de la academia puertorriqueña de la Lengua Española, 7, pp. 129-143.

Salvador, G. (1964). «La fonética andaluza y su propagación social y geográfica», Presente y futuro de la lengua española, II. Madrid: Instituto de Cultura Hispánica, pp. 183-188.

Samper Padilla, J. A. (1990). Estudio sociolingüístico del español de Las Palmas de Gran Canaria. Las Palmas de Gran Canaria: Caja de Ahorros de Canarias.

Terrell, T. (1975). «La nasal implosiva y final en el español de Cuba», Anuario de Letras, 19, pp. 257-271.

Trujillo, R. (1980). Resultados de dos encuestas dialectales en Masca. La Laguna: Instituto de Estudios Canarios.

Vida Castro, M.A. (2004). Estudio sociofonológico del español hablado en la ciudad de Málaga. Alicante: Publicaciones de la Universidad de Alicante.

Vida Castro, M. A. (2007). El español hablado en Málaga. Sarriá: Málaga.

Villena Ponsoda, J. A. y J. A. Moya Corral (2016). «Análisis comparativo de un cambio fonológico erosivo. Variación de /d/ intervocálica en dos comunidades de habla (Granada y Málaga)». Boletín de Filolofía, LI (2), pp. 281-321

Zmijanac, S. (2012). «Estudio sociolingüístico y foneticoacústico de la variación de la obstruyente ve$\operatorname{lar} / \mathrm{x} /$ en el español hablado en Málaga». En J. A. Villena Ponsoda y A. M. Ávila Muñoz, Estudios sobre el español de Málaga. Pronunciación, vocabulario y sintaxis. Málaga: Sarriá. 